Hydrological Research Letters, 4, 20-24 (2010)

Published online in J-STAGE (www.jstage.jst.go.jp/browse/HRL). DOI: 10.3178/HRL.4.20

\title{
Reproducibility of Regional-Mean Probable Precipitation by High-Resolution Climate Models
}

\author{
Toshiyuki Kitajima ${ }^{1}$, Kazuyoshi Yoshimatsu ${ }^{1}$, Hiroshi Ohno ${ }^{1}$ and Koji Ishihara ${ }^{2}$ \\ ${ }^{1}$ Japan Meteorological Agency, Tokyo, Japan \\ ${ }_{2}^{2}$ Meteorological Research Institute, Tsukuba, Japan
}

\begin{abstract}
:
We assessed the reproducibility of regional-mean probable precipitation in Japan estimated based on the results of three runs of a $20 \mathrm{~km}-\mathrm{mesh}$ global climate model (MRIGCM20) and one run of a $20 \mathrm{~km}-\mathrm{mesh}$ regional climate model (MRI-RCM20). These models inappropriately simulate the annual maximum daily precipitation at individual grid points, especially in relation to the spatial pattern, but estimate the regional mean reasonably well. MRI-GCM20 runs simulated data over annual maximum regional-mean 10-day precipitation better than 1-day. Precipitation and return values estimated by MRI-GCM20 were quite different from those by MRI-RCM20. Among the three MRI-GCM20 runs, one forced by SST without year-to-year variability estimated the standard deviation of the annual maximum precipitation as smaller than those forced by SST with year-to-year variability. The reproducibility of the average and standard deviation of the annual maximum precipitation is correlated with the reproducibility of 30-year return values.
\end{abstract}

KEYWORDS high-resolution climate global model; regional climate model; extremes; probable precipitation; annual maximum precipitation

\section{INTRODUCTION}

Increasing extreme weather events is one of the most important concerns, so the Japan Meteorological Agency (JMA) published the Climatic Risk Map of Extreme Events (JMA, 2007; hereafter Riskmap07) on the JMA web site. Riskmap07 presents probable precipitation based on observed precipitation at observatories in Japan and indicates the present risk of heavy rainfall.

In recent years, the Meteorological Research Institute (MRI) has developed several high-resolution climate models to project future climate change. The $20 \mathrm{~km}$-mesh global climate model (MRI-GCM20) and the $20 \mathrm{~km}-\mathrm{mesh}$ regional climate model (MRI-RCM20) were confirmed to accurately reproduce the present climate around Japan, including average temperature and annual precipitation (Mizuta et al., 2006; Kurihara et al., 2005; JMA, 2005b).

Some studies estimate future changes in extreme events and present several suggestions for estimating probable precipitation using these climate models. Wada et al. (2006) found that it was necessary that the assessment of probable precipitation using MRI-RCM20 be based on values averaged over a region of about 60 kilometers square in extent. In contrast, Wada et al. (2008) showed that it was possible to assess the reproducibility of the probable precipitation at individual grid points with MRI-GCM20. In general, however, the smallest scale of weather events that can be represented by a climate model is a few times the horizontal resolution of the model.

As for the time scale, Fowler et al. (2007) indicated that models they used underestimated return values of 1-day precipitation in the UK but reasonably simulate longerduration (5- or 10-day) extremes. They studied 1,2, 5 and 10-day precipitation totals. In their paper, results have been presented for only 1 - and 10-day totals, because they said that 1 - and 10-day totals represented short and long-duration precipitation events respectively.

We focused on the regional mean of precipitation totals, and checked their reproducibility by MRI-GCM20 and MRIRCM20. We showed results of 1- and 10-day precipitation similar to Fowler et al. (2007).

\section{DATA}

\section{Models}

Two models (MRI-GCM20 and MRI-RCM20) were used in this study (Table I). MRI-GCM20 is a $20 \mathrm{~km}$-mesh global climate model (Mizuta et al., 2006; Noda et al., 2007) developed by MRI under the "Kyousei Project." We used three runs of MRI-GCM20 that had been integrated for 20 years or more (AM, AT, AJ). These experiments are forced with different sea-surface temperatures (SSTs). The SST for $\mathrm{AM}$ is the result of an MRI Coupled General Circulation Model (CGCM) run and is warmer around northern Japan and colder in the south of Japan than the observed SST. AJ and AT are prescribed by the observed SSTs. The SST for $\mathrm{AJ}$ is the 12-year mean without year-to-year variability. MRI-RCM20 is a $20 \mathrm{~km}-\mathrm{mesh}$ regional climate model (Kurihara et al., 2005; JMA, 2005b) developed by MRI. Although the integration period of MRI-RCM20 slightly differs from that of MRI-GCM20, this is not likely to have influenced our results.

\section{Observations}

To assess the reproducibility of the models, we used daily precipitation data observed at 151 observatories in Japan from 1979 to 1998 and at AMeDAS (Automated Meteorological Data Acquisition System) stations from 1981 to 2000 (only for assessing annual mean daily precipitation).

Correspondence to: Toshiyuki Kitajima, Japan Meteorological Agency, 1-3-4 Otemachi, Chiyoda-ku, Tokyo 100-8122, Japan, E-mail: kitajimatoshiyuki@met.kishou.go.jp C2010, Japan Society of Hydrology and Water Resources. 
Table I. Models used in this study

\begin{tabular}{lllccccc}
\hline \multirow{2}{*}{ Model Acronym } & Region & $\begin{array}{c}\text { Horizontal } \\
\text { Resolution }\end{array}$ & Driving Data & $\begin{array}{c}\text { Prescribed } \\
\text { SST }\end{array}$ & $\begin{array}{c}\text { Year-to-year } \\
\text { Variability }\end{array}$ & $\begin{array}{c}\text { Integration } \\
\text { Period }\end{array}$ \\
\hline \multirow{2}{*}{ MRI-GCM20 } & AM & Global & $20 \mathrm{~km}$ & - & Yes & CGCM2.3.2 & 1979 to 1998 \\
\cline { 2 - 8 } & AT & Global & $20 \mathrm{~km}$ & - & Yes & Observation & 1979 to 1998 \\
\cline { 2 - 8 } & AJ & Global & $20 \mathrm{~km}$ & - & No & Observation & 20 years \\
\hline MRI-RCM20 & & Around Japan & $20 \mathrm{~km}$ & CGCM2.0 & CGCM2.0 $0^{\mathrm{a}, \mathrm{c}}$ & Yes & 1981 to 2000 \\
\hline
\end{tabular}

a CGCM: MRI Coupled General Circulation Model (Yukimoto et al., 2001; Yukimoto et al., 2006).

b SST for MRI-GCM20-AJ is the 12-year mean from 1982 to 1993.

c SST for MRI-RCM20 was adjusted so that the 20-year average of daily SST was equal to the 20-year average of the observed SST for each day (Kurihara et al., 2005).

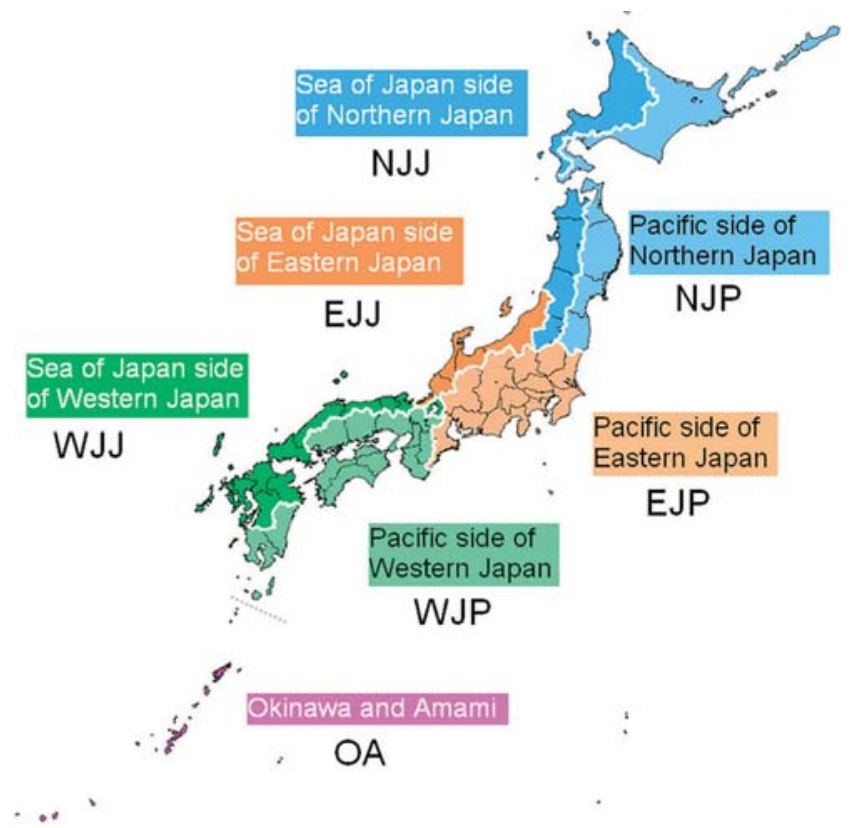

Figure 1. Seven regions defined for the seasonal forecast of JMA.

\section{METHODOLOGY}

The 10-day precipitation is the sum of the daily precipitation in any continuous period of 10 days. We defined the periods of our 10-day precipitation data starting with January 1 to 10 and running through December 22 to 31 for every year.

The regional mean is defined as the average of the data within each region defined for the seasonal forecast by JMA (Figure 1) in this study. The model's grid-point values over land nearest to the observatories are used. It must be noted that, in this study, the annual maximum regional-mean value refers to the annual maximum value of the regional averaged precipitation, not the regional average of annual maximum precipitation at each observatory or grid point.

Moreover, $R 1$ denotes the daily precipitation, and $R 10$ the 10 -day precipitation. The regional means of $R 1$ and $R 10$ are referred to as $\overline{R 1}$ and $\overline{R 10}$. The annual maxima of $R 1, R 10, \overline{R 1}$ and $\overline{R 10}$ are called $R 1_{\max }, R 10_{\max }, \bar{R} 1_{\max }$ and $\overline{R 10}_{\max }$.

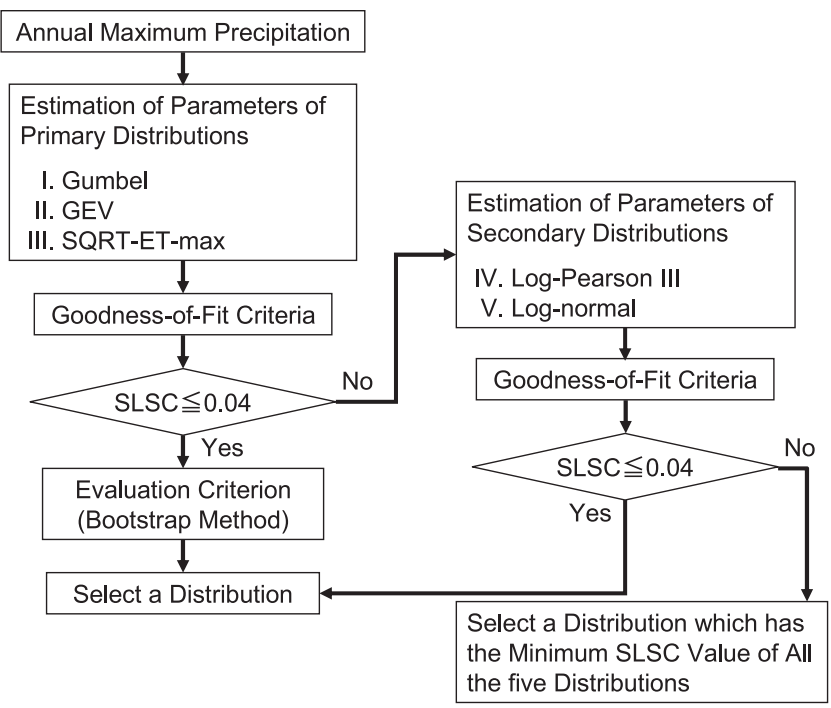

Figure 2. Flow chart of algorithm for selecting extreme value distributions. SLSC is "Standard Least-Squares Criterion" defined by Takara and Takasao (1988).

\section{Probable Precipitation}

The algorithm used for selecting extreme value distributions fitting the annual maximum precipitation is illustrated in Figure 2. The algorithm is based on that used for Riskmap07 but has been partially modified. We used the bootstrap resampling method as the evaluation criterion for extreme value distributions instead of the jackknife procedure in the algorithm of Riskmap07, in order to get a number of probable precipitation samples large enough to estimate a confidence interval. The resampling was repeated 1000 times. The 1000 probable precipitations were made by applying each resample data set to the selected distribution. The range between 5 th and 95 th percentiles of the probable precipitation is considered to be the $90 \%$ confidence interval in this study.

\section{RESULTS}

\section{Average and Frequency of Precipitation}

MRI-GCM20 and MRI-RCM20 generally reproduce the spatial pattern of the annual average precipitation observed by AMeDAS (Figures 3 and S1). All of the MRI-GCM20 runs underestimate the precipitation on the Pacific side of 
Japan, but MRI-RCM20 overestimates it. The boundary condition of MRI-RCM20 is supposed to contribute to inadequate precipitation especially in Northern Japan (JMA, 2005b). MRI-GCM20 also underestimates the frequency of days with precipitation of $50 \mathrm{~mm}$ or more, but MRI-RCM20 overestimates it (Figure S2).

\section{Comparison of Regional-mean Value with Grid-Point Value}

Figure 4 illustrates the 20 -year means of $R 1_{\text {max }}$ and $\overline{R 1}_{\max }$ of the observation and MRI-GCM20-AT (see also Figure S3). Figure $4 \mathrm{a}$ indicates that the model underestimates the daily precipitation all over Japan, particularly around western Japan. The spatial pattern of $R 1_{\max }$ seems to suggest a problem in that the difference of $R 1_{\max }$ produced by the model between the northern part and the southern part is smaller than that of the observation. In the case of $\overline{R 1}_{\text {max }}$ (Figure $4 \mathrm{~b}$ ), the spatial pattern is relatively accurate when compared to $R 1_{\max }$, although the amount is underestimated.

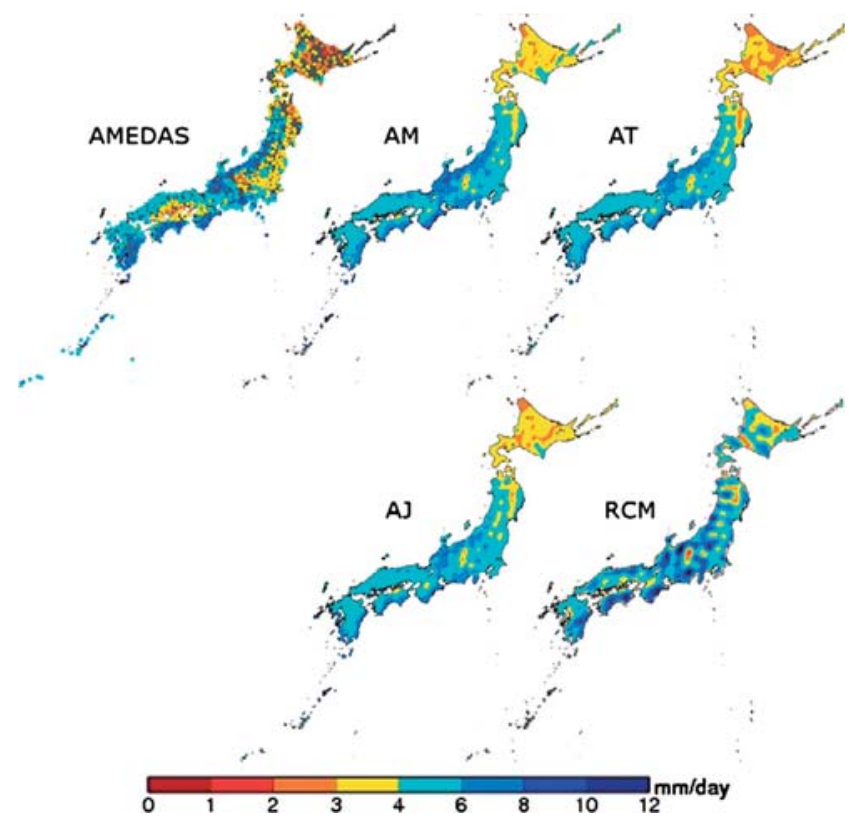

Figure 3. 20-year average of annual mean daily precipitation in Japan.

(a) R1

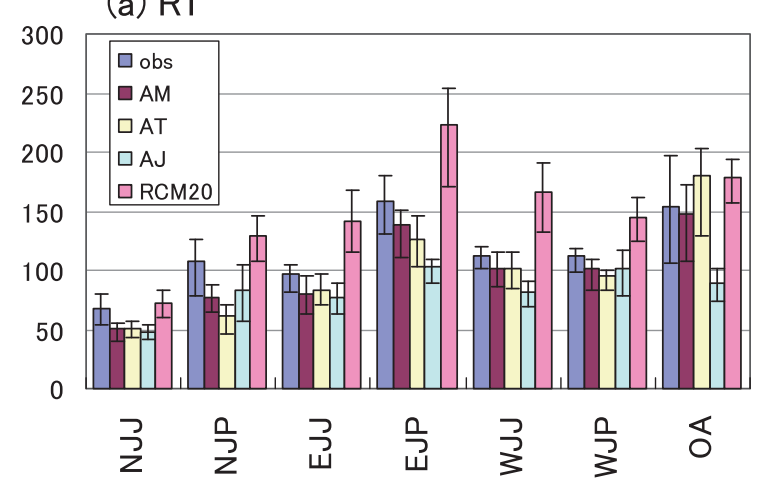

MRI-GCM20-AM and-AJ exhibit the same tendency as AT. MRI-RCM20 simulates the regional mean better as well, but the amount is overestimated (not shown).

\section{Annual Maximum Regional-Mean Precipitation}

The models basically reproduce the 20 -year means of $\overline{R 1}_{\text {max }}$ and $\overline{R 10}_{\text {max }}$ (Figure S4). For $\overline{R 1}_{\text {max }}$, the difference between the models and the observations is about $25 \%$. The difference among the MRI-GCM20 runs is small relative to that between MRI-RCM20 and MRI-GCM20. In the case of MRI-GCM20, $\overline{R 10}_{\text {max }}$ is generally reproduced better than $\overline{R 1}_{\text {max }}$. MRI-RCM20, however, tends to overestimate $\overline{R 10}_{\text {max }}$ more than $\overline{R 1}_{\text {max }}$.

The standard deviation of $\overline{R 1}_{\text {max }}$ and $\overline{R 10}_{\text {max }}$ is reproduced worse by the models when compared to the 20 -year mean. The standard deviation by MRI-GCM20-AJ is smaller than that of the other MRI-GCM20 runs (Figures 6 and S4). This is likely to have been caused by use of the SST without year-to-year variability for this run.

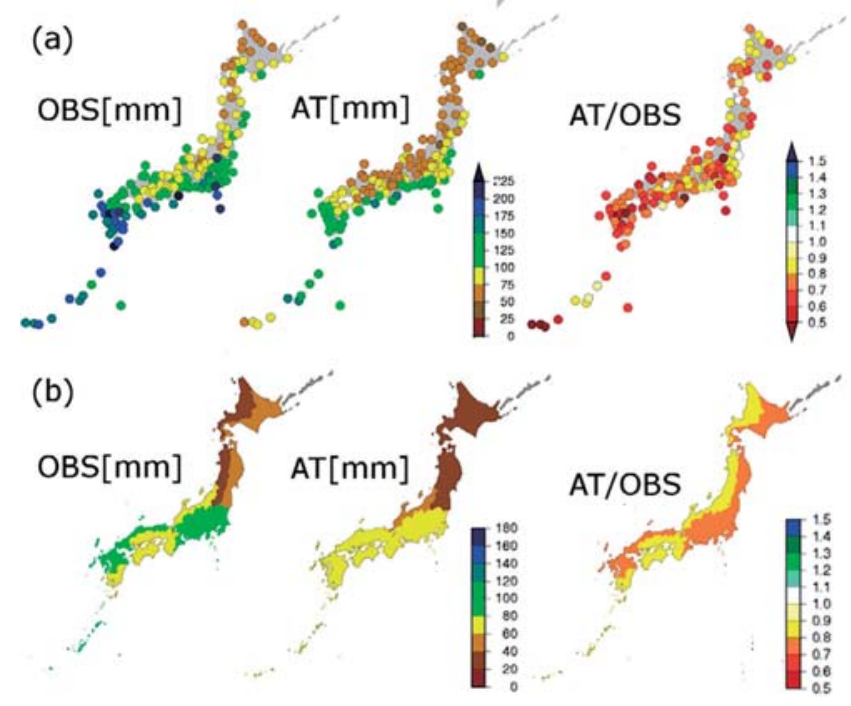

Figure 4. 20-year average of annual maximum precipitation of the model (MRI-GCM20-AT) and the observation. (a) Comparison at stations and grid points. (b) Comparison by region.

(b) R10

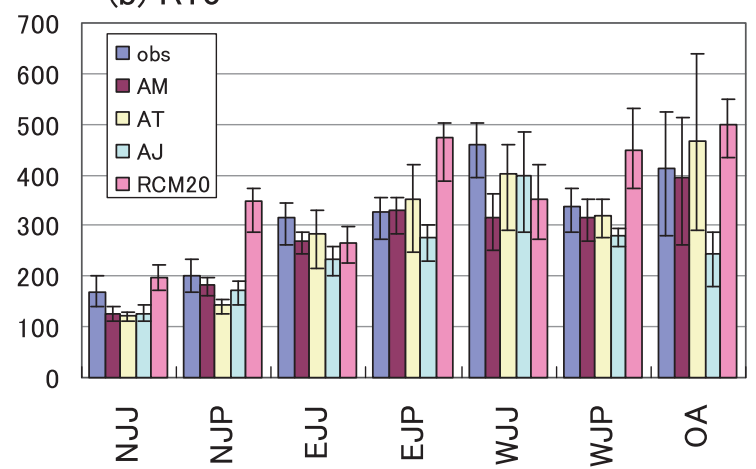

Figure 5. Probable precipitation (30-year return value) [mm] for regions. (a) 1-day precipitation. (b) 10-day precipitation. The error bars represent the $90 \%$ confidence intervals estimated using the bootstrap method. 

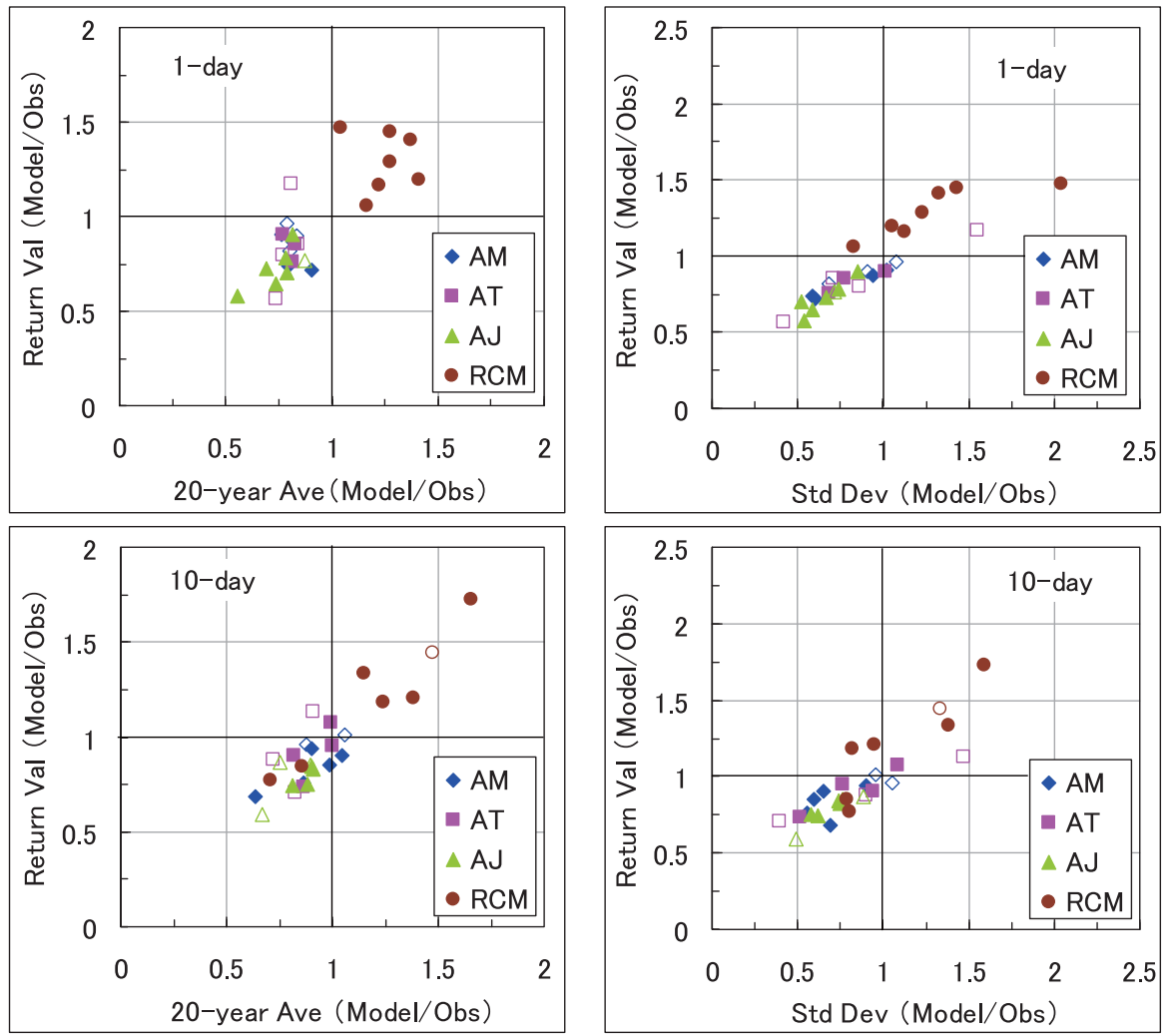

Figure 6. Scatter plots of the model-to-observation ratios of the probable precipitation (return value) and the 20 -year average (left) and standard deviation (right) of the regional-mean annual maximum precipitation. The open symbols represent regions in which the selected distribution for the model is the same type as that for the observation.

\section{Probable Precipitation}

The probable precipitation (30-year return value) of $\overline{R 1}$ and $\overline{R 10}$ is underestimated by MRI-GCM20 and overestimated by MRI-RCM20 (Figure 5). The differences among the MRI-GCM20 runs are small except for Okinawa and Amami, where the value of MRI-GCM20-AJ is smaller than that of the other runs.

The $90 \%$ confidence intervals estimated by the bootstrap method are $20 \%$ to $60 \%$ of the probable precipitation, so the errors are $10 \%$ to $30 \%$. The errors are thought to be basically due to the small sample size (Takara and Takasao, 1988).

The relation between the reproducibility of $\overline{R 1}_{\max }$ and $\overline{R 10}_{\max }$ and that of the 30 -year return value is depicted in Figure 6 . The reproducibility of the average and standard deviation of the annual maximum precipitation is correlated with the reproducibility of 30 -year return values for both $\overline{R 1}$ and $\overline{R 10}$, whether the types of the distributions of the observation and the models are the same or not.

\section{DISCUSSION AND CONCLUSIONS}

This study assessed the reproducibility of the regionalmean probable precipitation in Japan estimated from MRIGCM20 and MRI-RCM20. The results are summarized as follows:

- The models reproduce the spatial pattern of the annual maximum regional-mean daily precipitation $\left(\overline{R 1}_{\max }\right)$ better than that of grid-point values $\left(R 1_{\max }\right)$. It is supposed that $\overline{R 1}_{\text {max }}$ often occurs with larger scale events such as a typhoon or extratropical cyclone that can be reproduced by climate models better than smaller scale events.

- MRI-GCM20 simulated $\overline{R 10}_{\text {max }}$ better than $\overline{R 1}_{\text {max }}$, but MRI-RCM20 tended to overestimate $\bar{R}^{10}$ max more than $\overline{R 1}_{\max }$. It is consistent with the result in Fowler et al. (2007) that the reproducibility of longer-duration extremes is better than that of 1 day.

- There are smaller differences among the three runs of MRI-GCM20 than between MRI-RCM20 and MRIGCM20. MRI-GCM20-AJ, which is forced by the 12year mean SST without year-to-year variability, tends to have smaller standard deviations than the other MRIGCM20 runs.

This study demonstrated that the regional mean and 10day sum are likely to improve the reproducibility of the probable precipitation estimated by the model. We hereafter will estimate the change in probable precipitation using regional-mean 10-day values.

\section{ACKNOWLEDGEMENTS}

We used the MRI-GCM20 data created in the "Kyousei Project." This study was supported by the Special Research on Climate Change Projection of JMA conducted at MRI. 


\section{T. KITAJIMA ET AL.}

\section{SUPPLEMENTS}

Supplement 1 includes 4 figures.

Figure S1. Enlargement of Figure 3.

Figure S2. Frequency of daily precipitation intensity.

Figure S3. Enlargement of Figure 4.

Figure S4. 20-year Average and Standard Deviation of Annual Maximum Regional-Mean Precipitation.

\section{REFERENCES}

Fowler HJ, Ekström M, Blenkinsop S, Smith AP. 2007. Estimating change in extreme European precipitation using a multi-model ensemble. Journal of Geophysical Research. 112: D18104. doi:10.1029/2007JD008619.

Japan Meteorological Agency. 2005a. Report on Climate Change 2005. http://www.data.kishou.go.jp/climate/cpdinfo/climate change/ 2005/pdf/2005_all.pdf. [February 28, 2009] (in Japanese).

Japan Meteorological Agency. 2005b. Global Warming Projection 6. http://ds.data.jma.go.jp/tcc/tcc/products/gwp/gwp6/index.html. [February 28, 2009]

Japan Meteorological Agency. 2007. Climatic Risk Map of Extreme Events. http://www.data.kishou.go.jp/climate/riskmap/ index.html. [February 28, 2009](in Japanese).

Kurihara K, Ishihara K, Sasaki H, Fukuyama Y, Saitou H, Takayabu I, Murazaki K, Sato Y, Noda A. 2005. Projection of Climatic Change over Japan Due to Global Warming by HighResolution Regional Climate Model in MRI. SOLA 1: 97100. doi:10.2151/sola.2005-026.

Mizuta R, Oouchi K, Yoshimura H, Noda A, Katayama K, Yukimoto S, Hosaka M, Kusunoki S, Kawai H, Nakagawa
M. 2006. 20-km-Mesh Global Climate Simulations Using JMA-GSM Model-Mean Climate States-. Journal of the Meteorological Society of Japan 84: 165-185. doi:10.2151/ jmsj.84.165.

Noda A, Kusunoki S, Nakamura M. 2007. Development of Super High Resolution Climate Models. Annual Report of the Earth Simulator Center April 2006-March 2007: 271-279.

Takara K, Takasao T. 1988. Criteria for Evaluating probability Distribution Models in Hydrologic Frequency Analysis. Journal of Hydraulic, Coastal and Environmental Engineering 393(II-9): 151-160 (in Japanese with English abstract).

Wada K, Kawasaki M, Tomizawa Y. 2006. Study on the Flood Risk Assessment of Global Warming by Regional Climate Model. Annual Journal of Hydraulic Engineering 50: 613618 (in Japanese with English abstract).

Wada K, Kawasaki S, Tomizawa Y, Kusunoki S, Kurihara K. 2008. Study on the Flood Risk Assessment of Global Warming by Regional Climate Model and High Resolution General Circulation Model. Journal of Japan Society of Hydrology and Water Resources 21: 12-22. doi:10.3178/jjshwr.21.12 (in Japanese with English abstract).

Yukimoto S, Noda A, Kitoh A, Sugi M, Kitamura Y, Hosaka M, Shibata K, Maeda S, Uchiyama T. 2001. The New Meteorological Research Institute Coupled GCM (MRICGCM2)-Model Climate and Variability-. Papers in Meteorology and Geophysics 51: 47-88.

Yukimoto S, Noda A, Kitoh A, Hosaka M, Yoshimura H, Uchiyama T, Shibata K, Arakawa O, Kusunoki S. 2006. Present-Day Climate and Climate Sensitivity in the Meteorological Research Institute Coupled GCM Version 2.3 (MRI-CGCM 2.3). Journal of the Meteorological Society of Japan 84: 333363. doi:10.2151/jmsj.84.333. 